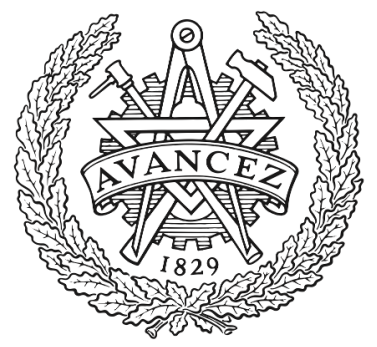

CHALMERS

UNIVERSITY OF TECHNOLOGY

\title{
All-Polymer Conducting Fibers and 3D Prints via Melt Processing and Templated Polymerization
}

Downloaded from: https://research.chalmers.se, 2023-04-26 15:20 UTC

Citation for the original published paper (version of record):

Hofmann, A., Östergren, I., Kim, Y. et al (2020). All-Polymer Conducting Fibers and 3D Prints via Melt Processing and Templated Polymerization. ACS Applied Materials \& Interfaces, 12(7):

8713-8721. http://dx.doi.org/10.1021/acsami.9b20615

N.B. When citing this work, cite the original published paper. 


\section{All-Polymer Conducting Fibers and 3D Prints via Melt Processing and Templated Polymerization}

Anna I. Hofmann, ${ }^{*} \S$ Ida Östergren, ${ }^{\S}$ Youngseok Kim, Sven Fauth, Mariavittoria Craighero, Myung-Han Yoon, Anja Lund, and Christian Müller*

Cite This: ACS Appl. Mater. Interfaces 2020, 12, 8713-8721

Read Online

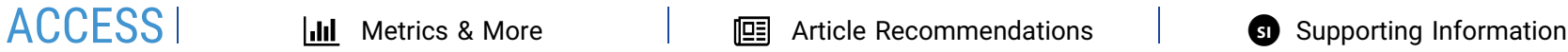

ABSTRACT: Because of their attractive mechanical properties, conducting polymers are widely perceived as materials of choice for wearable electronics and electronic textiles. However, most state-of-the-art conducting polymers contain harmful dopants and are only processable from solution but not in bulk, restricting the design possibilities for applications that require conducting micro-to-millimeter scale structures, such as textile fibers or thermoelectric modules. In this work, we present a strategy based on melt processing that enables the fabrication of nonhazardous, all-polymer conducting bulk structures composed of poly(3,4-ethylenedioxythiophene) (PEDOT) polymerized within a Nafion template. Importantly, we employ classical polymer processing techniques including melt extrusion followed by fiber spinning or fused filament 3D printing, which cannot be implemented with the majority of doped polymers. To demonstrate the versatility of our approach, we fabricated melt-spun PEDOT:Nafion fibers, which are highly flexible, retain their conductivity of about 3 $\mathrm{S} \mathrm{cm}^{-1}$ upon stretching to $100 \%$ elongation, and can be used to construct organic electrochemical transistors (OECTs). Furthermore, we demonstrate the precise 3D printing of complex conducting structures from OECTs to centimeter-sized PEDOT:Nafion figurines and millimeter-thick 100-leg thermoelectric modules on textile substrates. Thus, our strategy opens up new possibilities for the design of conducting, allpolymer bulk structures and the development of wearable electronics and electronic textiles.

KEYWORDS: melt processing, conducting elastic PEDOT:Nafion fibers, 3D printing, organic electrochemical transistors (OECTs), electronic textiles

\section{INTRODUCTION}

Conducting polymers constitute a class of materials that is of considerable interest for a large variety of applications, ranging from thermoelectrics ${ }^{1,2}$ to electronic textiles (e-textiles), ${ }^{3-5}$ electronic skin (e-skin), ${ }^{6,7}$ and bioelectronics. ${ }^{8,9}$ In order to realize self-supporting components such as conducting fibers ${ }^{5}$ or the legs of thermoelectric devices, ${ }^{10-13}$ supple bulk materials with micro-to-millimeter dimensions are needed. To facilitate the efficient fabrication of such thick bulk structures, it would be advantageous if the conducting polymers could be transformed using established polymer processing methods such as melt extrusion, melt spinning, and fused filament fabrication (FFF), a common 3D printing method.

As a matter of fact, many conjugated polymers such as polythiophenes, poly(phenylenevinylene)s, and polyfluorenes are melt processable in their undoped form and can be rendered conducting through sequential doping with small molecule dopants. ${ }^{14-16}$ However, sequential doping requires diffusion of the dopant molecule into the polymer, which becomes rate limiting if thick materials are to be doped, unless porous structures are used. ${ }^{17}$ On the other hand, there are a few examples for conjugated polymers that are melt processable in their doped form, one of them being polyaniline doped with dodecylbenzenesulfonic acid (DBSA). ${ }^{18-20} \mathrm{Un}$ fortunately, the strong acid dopant is corrosive and can be expected to damage the internal steel surfaces of extruders and FFF printers. $^{21}$

Aside processability aspects, one has to take into account that the conducting polymers must be benign and chemically stable in air and water in order to be viable for wearable electronics. While conjugated polymers themselves are considered biocompatible, most of the currently studied conducting polymer systems contain harmful acid or redox dopants, which may be released over time because the doping process is reversible. $^{22,23}$ Similar problems can be anticipated for melt-processable polymer nanocomposites, which may leak nanofillers, such as carbon nanotubes, graphene, or metal nanoparticles. $^{24}$

Received: November 20, 2019

Accepted: January 27, 2020

Published: February 11, 2020 
(a) Nafion Precursor

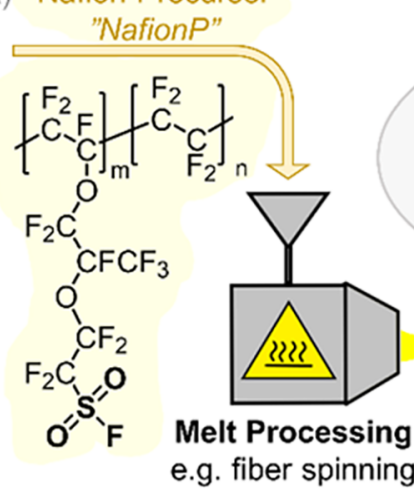

(b)
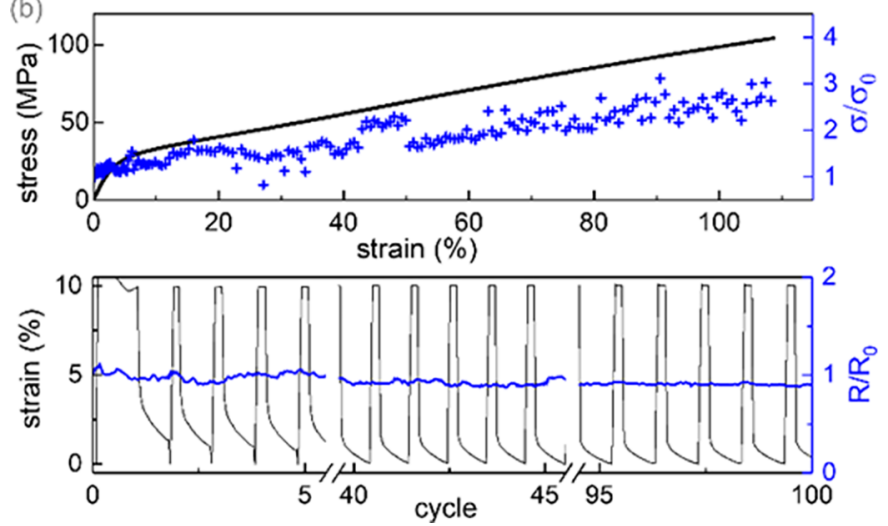
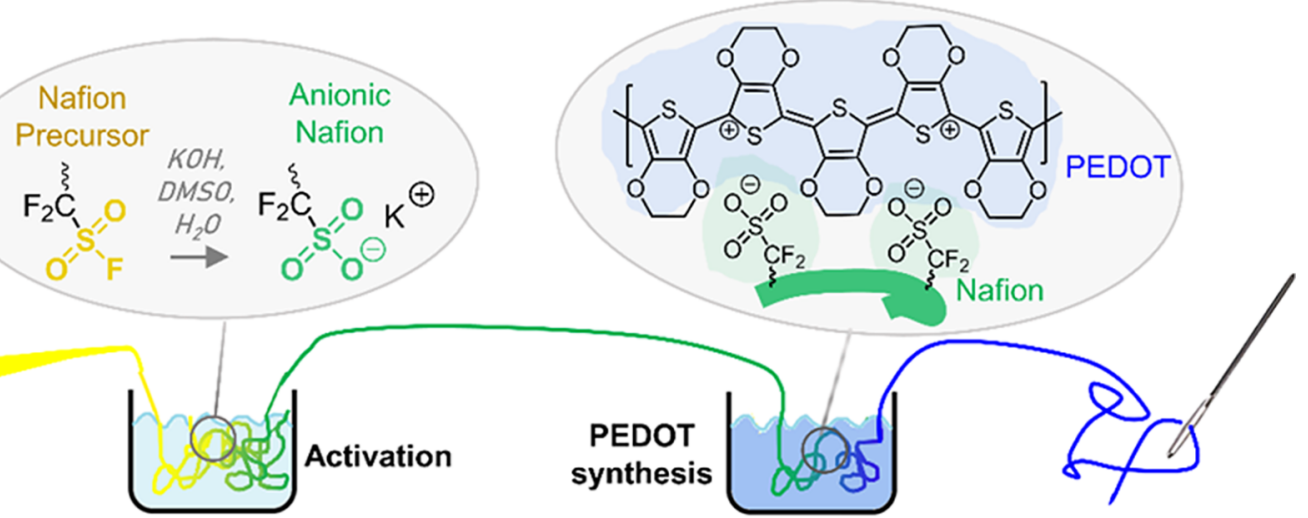
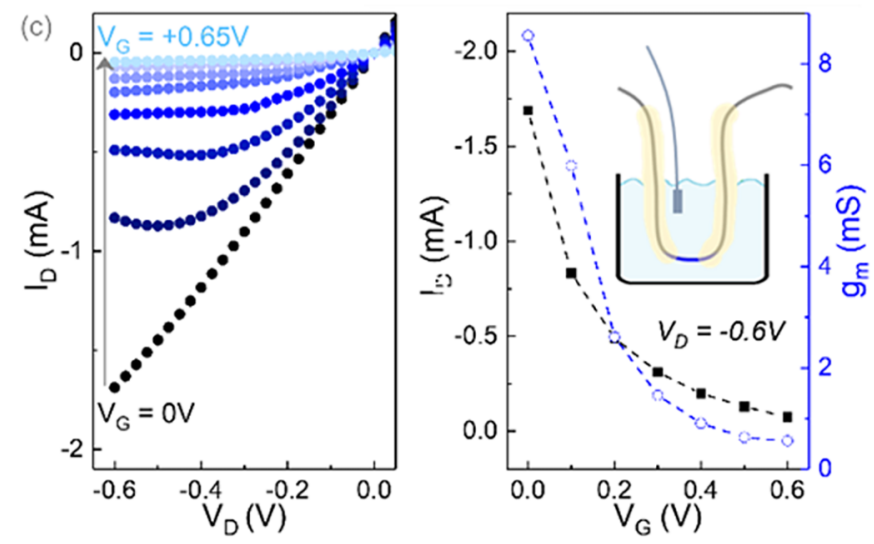

Figure 1. (a) Chemical structures and the processing scheme for the Nafion precursor, activated Nafion, and PEDOT. (b) Evolution of the electrical conductivity of PEDOT:Nafion fibers upon mechanical stretching until break (top) and upon cyclic deformation within the elastic regime (bottom). (c) Output characteristics $\left(I_{\mathrm{D}}\right.$ vs $\left.V_{\mathrm{D}}\right)$, transfer curve $\left(I_{\mathrm{D}}\right.$ vs $\left.V_{\mathrm{G}}\right)$, and transconductance $g_{\mathrm{m}}$ of a PEDOT:Nafion fiber OECT operating in $0.1 \mathrm{M} \mathrm{NaCl}$ electrolyte and with an $\mathrm{AgCl}$ pellet gate electrode.

One way to design a chemically stable doped polymer complex and eliminate risks related to the leakage of harmful substances is the use of an all-polymer system, for example, a conjugated polymer doped with a polymeric counter ion. A prominent example for such a conjugated polymer:polyanion complex is poly(3,4-ethylenedioxythiophene):poly(styrene sulfonate) (PEDOT:PSS), which finds widespread use in a myriad of applications; thanks to its high conductivity, excellent chemical stability, biocompatibility, as well as its straight-forward and cost-efficient synthesis. ${ }^{25,26}$ However, PEDOT:PSS is only processable from an aqueous dispersion, which limits its use to certain bulk processing methods, such as the solution-casting of tapes ${ }^{27-29}$ or wet-spinning of fibers, ${ }^{30-35}$ some of which reach conductivities of more than $10^{3} \mathrm{~S} \mathrm{~cm}^{-1}$. Also, blends of PEDOT:PSS and insulating polymers, which display promising electrical and mechanical properties, are mostly processed from solution. ${ }^{36-38}$ More recently, it has been shown that PEDOT can also be complexed with a large variety of other polymeric counter ions, ${ }^{39,40}$ ranging from polyanionic polysaccharides ${ }^{41-43}$ to fluoropolymers such as Nafion, ${ }^{44-48}$ a term commonly used to describe sulfonated tetrafluoroethylene-based copolymers. However, all of these PEDOT complexes are intractable without the use of a solvent and cannot be processed from the melt, which prevents the use of PEDOT for most bulk processing methods that are widely used in polymer technology.

Thus, we set out to develop a novel strategy that permits the fabrication of an all-polymer PEDOT-based bulk material, combining classical melt processing methods with the templated oxidative polymerization of 3,4-ethylenedioxythiophene (EDOT). We chose to work with a thermoplastic fluoropolymer that carries sulfonyl fluoride groups, that is, a Nafion precursor, which can be melt processed into any desired shape. Upon chemical activation, the precursor bulk is transformed into anionic Nafion, which serves as a bulk template during the oxidative dispersion polymerization of EDOT. The obtained PEDOT:Nafion complex is flexible and highly stretchable and shows a bulk conductivity of about $3 \mathrm{~S}$ $\mathrm{cm}^{-1}$. To illustrate the versatility of our strategy, we prepared conducting fibers, fabricated organic electrochemical transistors (OECTs) and 3D printed different complex bulk structures, including a 100-leg thermoelectric module on a textile substrate.

\section{RESULTS AND DISCUSSION}

We identified Nafion as a suitable candidate for our work because it can serve as a bulk template for PEDOT and shows excellent biocompatibility. ${ }^{49}$ The PEDOT:Nafion bulk materials were obtained via a three-step process, consisting of (1) melt processing of the Nafion precursor into the desired shape and (2) an activation step yielding the anionic Nafion, followed by (3) the synthesis of PEDOT within the Nafion template (Figure 1a).

To prepare conducting fibers, the Nafion precursor was melt-spun into fibers with a diameter of approximately $200 \mu \mathrm{m}$. The precursor fibers were then soaked in an activation bath of $\mathrm{H}_{2} \mathrm{O} / \mathrm{DMSO} / \mathrm{KOH}$, following a well-established activation 
procedure. $^{50-53}$ As confirmed by elemental analysis and infrared spectroscopy (Figure S1 and Table S1), ${ }^{52}$ the activation process transformed the sulfonyl fluoride groups into sulfonic acid groups, resulting in an ionic Nafion with increased mechanical stiffness that swells in water. After washing it in water, the activated Nafion was immersed into an aqueous dispersion of EDOT that penetrated and swelled the anionic Nafion bulk. Upon addition of the oxidants, the EDOT monomer polymerized within the Nafion template. In this way, we were able to produce several meters of PEDOT:Nafion fibers in a synthesis bath with a volume smaller than $10 \mathrm{~mL}$ (Figure 2), a process reminiscent of traditional batch dyeing

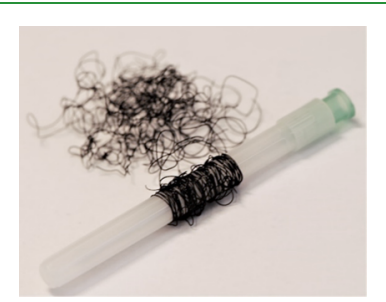

Figure 2. Photograph of melt-spun PEDOT:Nafion fibers, wrapped around a $5 \mathrm{~mm}$ wide syringe cover.

used in the textile industry. The resulting fibers were dark blue and flexible and displayed a bulk conductivity of $\sigma_{\text {PEDOT:Nafion }}=$ $3 \pm 1 \mathrm{~S} \mathrm{~cm}^{-1}$. It is noteworthy that the measured conductivity is comparable to the conductivity of melt extruded PANI:DBSA ${ }^{19}$ as well as most PEDOT:biopolymer complexes $^{40}$ and is higher than the conductivity of pristine PEDOT:PSS processed without cosolvents. ${ }^{54}$ Furthermore, PEDOT:Nafion displayed a Seebeck coefficient of $\alpha_{\text {PEDOT:Nafion }}$ $=12 \pm 2 \mu \mathrm{V} \mathrm{K}^{-1}$, indicating that the PEDOT within the complex is highly doped. In order to check the stability over time, we remeasured three different sample batches one year after preparation and found that their conductivity was unchanged (Figure S2). Elemental analysis revealed that the purified bulk material contained approximately one EDOT repeating unit per repeating unit of Nafion and that only small amounts of ions $(<1 \mathrm{wt} \%)$ are present within the complex (Table S1). This translates to a concentration of 12 wt \% PEDOT within the PEDOT:Nafion bulk, as compared to as much as $30 \mathrm{wt} \%$ in commercial bench-mark PEDOT:PSS.

To elucidate the nanostructure of PEDOT:Nafion, we analyzed the complex by X-ray scattering. The structure of Nafion is often described as a polytetrafluoroethylene (PTFE)like bulk, which is traversed by a hydrophilic network of nanochannels or nanoclusters, formed by the sulfonate side groups. It has been reported that this nanostructure gives rise to a peak at around $q \approx 2.0 \mathrm{~nm}^{-1}(d \approx 3.1 \mathrm{~nm})$ in small angle X-ray scattering (SAXS), ${ }^{55-57}$ which we also observe for our Nafion material after the melt processing and the activation step (Figure S3). In contrast, after the PEDOT synthesis, SAXS diffractograms of the resulting bulk PEDOT:Nafion did not display any distinct peak in this $q$ range (Figure S3). Thus, we argue that the PEDOT penetrates the hydrophilic nanochannels and disrupts the initial nanostructure of the Nafion bulk. Wide angle X-ray scattering (WAXS) diffractograms of PEDOT:Nafion displayed a broad scattering peak between $q \approx 12$ to $13 \mathrm{~nm}^{-1}(d \approx 0.5 \mathrm{~nm})$, which we attribute to the amorphous PTFE-like bulk (Figure S3). The absence of scattering features at around $q \approx 18 \mathrm{~nm}^{-1}(d \approx 0.35 \mathrm{~nm})$ is a sign of a low degree of $\pi$-stacking of the PEDOT chains.
We went on to characterize the mechanical properties of the complex and found that PEDOT:Nafion fibers are highly stretchable and display a Young's modulus $E \approx 620 \pm 100$ $\mathrm{MPa}$ and an elongation at break of more than $100 \%$. Further, the fibers are highly fatigue resistant and can be subjected to at least 100 strain-release cycles with $10 \%$ maximum strain without compromising their electrical conductivity (Figure 1b). A direct comparison with pristine-activated Nafion fibers, which are equally elastic (Figure S4), suggests that Nafion is the continuous, loadbearing phase that is impregnated by PEDOT-rich domains. Upon stretching, we observed a reversible increase of the conductivity, opposite to the behavior commonly observed for composite materials made from conducting fillers in an insulating matrix. We argue that upon stretching, PEDOT-rich domains are elongated, which leads to an improved percolation and a reversible increase in the conductivity of the material, similar to the behavior observed for PEDOT:PSS/polyurethane blends by Seyedin et al. $^{38}$

Because of their remarkable stretchability, PEDOT:Nafion fibers are of high interest for elastic interconnects in e-textiles and for the fabrication of wearable biosensors, ${ }^{6}$ such as sensors based on OECTs. In addition, anionic Nafion swells readily in water and is known for its excellent cation transport properties. ${ }^{44-46}$ Swelling tests on PEDOT:Nafion confirmed that the conducting bulk material retained a high degree of swellability and can take up over $10 \mathrm{wt} \%$ of water (Figure S5). Thus, we went on to study the performance of PEDOT:Nafion as a mixed ion-hole conductor in OECTs operating in the depletion mode. Devices made of a PEDOT:Nafion fiber $(\varnothing=$ $200 \mu \mathrm{m}$, channel length $\approx 1.5 \mathrm{~mm}$ ) displayed an ON/OFF ratio on the order of $10^{2}$ and a maximum transconductance $g_{\mathrm{m}}$ of $8.6 \mathrm{mS}$ at zero gate voltage $\left(V_{\mathrm{D}}=-0.6 \mathrm{~V}\right)$ (Figure $1 \mathrm{c}$ ). Hence, the measured transconductance is comparable to the $g_{\mathrm{m}}$ of microfabricated thin-film devices with channels made from PEDOT:PSS ${ }^{9}$ or other PEDOT-based materials. ${ }^{9,58}$ For PEDOT:PSS-based devices, it was observed that $g_{\mathrm{m}}$ increases with the channel thickness, as a result of the strong swelling and the constant volumetric capacitance $\left(C^{*}=39 \mathrm{~F} \mathrm{~cm}^{-3}\right)$ of PEDOT:PSS. ${ }^{59-61}$ Following the trend reported by Rivnay et al., ${ }^{61}$ PEDOT:PSS channels with dimensions comparable to our device can be expected to display a $g_{\mathrm{m}}$ of about $30-40 \mathrm{mS}$, which is four times higher than the transconductance measured for our device. For further elucidation, we determined the volumetric capacitance of PEDOT:Nafion by analysis of the gate current transient and by impedance spectroscopy and found a $C^{*}$ in the range of $9-15 \mathrm{~F} \mathrm{~cm}^{-3}$ (Figure S6). Thus, the lower $g_{\mathrm{m}}$ of our device can be related to a lower volumetric capacitance of PEDOT:Nafion as compared to PEDOT:PSS, which is also in agreement with the lower PEDOT concentration in PEDOT:Nafion. The response time of the PEDOT:Nafion OECTs was in the range of several seconds ( $\tau$ $\approx 18 \mathrm{~s}$ ), and cyclability of the devices confirmed that the electrical properties are stable upon immersion in water for at least $3 \mathrm{~h}$ (Figure S7).

In order to fabricate more diverse and complex bulk structures, we explored the transferability of our strategy to FFF 3D printing. For successful 3D printing, the thermoplastic polymer must display (1) good adhesion to the printing substrate and (2) sufficiently high stiffness to ensure correct feeding of the filament into the printer head. The Nafion precursor, however, does not adhere well to common print surfaces and is relatively soft $(E \approx 7.5 \mathrm{MPa})$. To overcome 

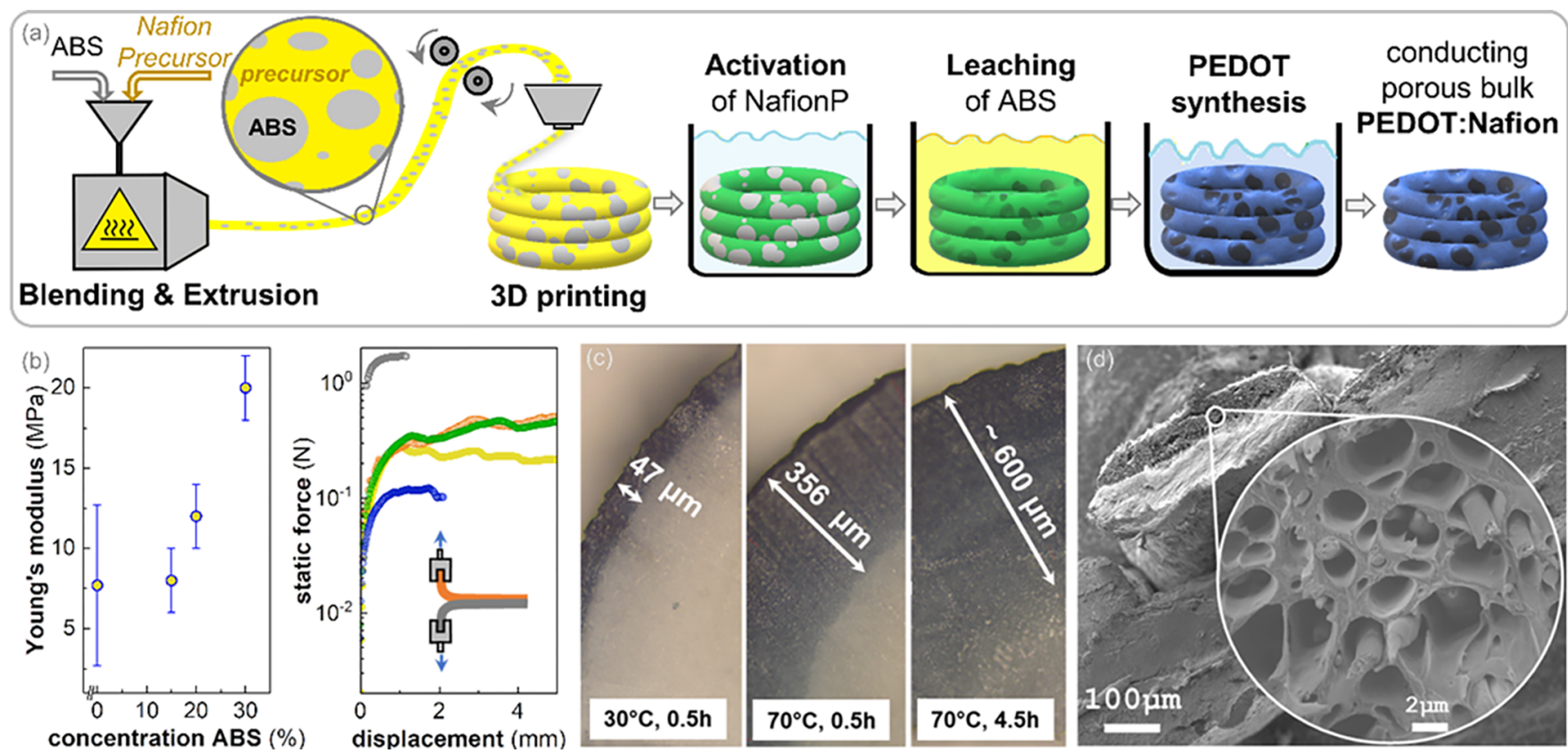

Figure 3. (a) Processing scheme for the FFF 3D printing of the precursor/ABS blend to obtain PEDOT:Nafion bulk structures. (b) Young's modulus of the Nafion precursor upon blending with ABS and the peeling test of ABS printed on HIPS (gray), Nafion/ABS blends on HIPS before activation (orange) and after activation (green), as well as Nafion/ABS on ABS before activation (yellow) and with a thin PEDOT:Nafion layer (blue). (c) Optical micrographs of cross sections of Nafion/ABS blends with PEDOT:Nafion layers of different thicknesses. (d) Cross section of a 3D-printed line of Nafion/ABS with a $30 \mu \mathrm{m}$ thick layer of porous PEDOT:Nafion, imaged by SEM.

these challenges, we developed a printable polymer blend of the Nafion precursor and an acrylonitrile-butadiene-styrene (ABS) plastic (Figure 3a). ABS, one of the benchmark materials for FFF 3D printing, displays a high stiffness at room temperature $(E \approx 1-3 \mathrm{GPa})$ and a processing temperature of $240{ }^{\circ} \mathrm{C}$. Scanning electron microscopy (SEM) images of the precursor/ABS blends depict a phase-separated system, corresponding to a precursor matrix interspersed with micrometer-sized ABS domains (Figure S8). Thus, the stiff ABS domains act as filler particles, which increase the stiffness of the blend. By screening different compositions of precursor/ ABS blends, we found that the stiffness of the blend increased significantly for $\mathrm{ABS}$ concentrations of more than $20 \mathrm{wt} \%$ (Figure $3 \mathrm{~b}$ ). Upon addition of 30 wt \% ABS, we obtained a precursor/ABS blend with a Young's modulus of $20 \mathrm{MPa}$. This content is high enough to allow for correct feeding of the filament into the printer head and for adherence to ABS, high impact polystyrene (HIPS), and textile substrates (Figure $3 \mathrm{~b}$ ). Thus, we chose to work with a precursor $/ 30$ wt \% ABS blend, which represents a good compromise between high stiffness and excessive content of the insulating ABS filler material.

We extruded the precursor/ABS blend (30 wt \% ABS) into filaments and printed various structures, which were then immersed into the activation bath (see scheme in Figure 3a). Because the activation is controlled by the diffusion of the activation solution into the precursor/ABS bulk, the thickness of the activated layer could be controlled by varying the temperature and the reaction time (Figure $3 \mathrm{c}$ ). We found that with increasing temperature, the activation reaction proceeded faster into the bulk, which we tentatively assign to improved swelling and diffusion of the alkaline solution into the material, as well as increased reaction kinetics. It should be noted that the thickness of the activated, anionic Nafion defines the thickness of the resulting conducting PEDOT:Nafion layer. Hence, a short activation of $30 \mathrm{~min}$ at room temperature, for instance, resulted in a PEDOT layer of 20-30 $\mu \mathrm{m}$. If the activation was allowed to proceed for several hours at $70{ }^{\circ} \mathrm{C}$, millimeter-thick samples were fully impregnated with PEDOT. Thus, very thick PEDOT:Nafion samples can be obtained by soaking the Nafion precursor in the activation solution for a prolonged time. In this work, we fabricated both micrometer thin conducting PEDOT:Nafion layers $\left(d_{\min } \approx 10 \mu \mathrm{m}\right)$ as well as millimeter thick conducting bulk structures $\left(d_{\max } \approx 7 \mathrm{~mm}\right)$, which opens up many new possibilities for the design of $3 \mathrm{D}$ printed structures.

In order to facilitate the diffusion of the EDOT monomer into the Nafion bulk, we chose to remove the ABS from the Nafion/ABS blend because it becomes redundant after printing. Upon leaching in a solution of $\mathrm{FeCl}_{3}$ in dimethyl sulfoxide (DMSO), we obtained a highly porous Nafion bulk (Figure 3d). At the same time, the leaching step introduced $\mathrm{Fe}^{3+}$ into the anionic Nafion. Because $\mathrm{Fe}^{3+}$ acts as the oxidant in the EDOT polymerization, no additional $\mathrm{FeCl}_{3}$ had to be added to initiate the polymerization reaction. The presence of $\mathrm{Fe}^{3+}$ within the Nafion helped to confine the polymerization locally, so that PEDOT was predominantly formed within the Nafion bulk and less in the water phase of the synthesis bath. The obtained porous PEDOT:Nafion bulk displayed a conductivity of $1-5 \mathrm{~S} \mathrm{~cm}^{-1}$ and a Seebeck coefficient of 10 $\pm 2 \mu \mathrm{V} \mathrm{K}^{-1}$, which is comparable to the electrical properties of PEDOT:Nafion processed without ABS.

We went on to test the electrochemical response of the printed material within OECT devices (Figure S9). In order to keep the channel dimensions similar to our fiber OECTs, we measured printed lines (width $1 \mathrm{~mm}$ ) with a $30 \mu \mathrm{m}$ thick, porous PEDOT:Nafion layer and a channel length of $1.5 \mathrm{~mm}$. The 3D printed devices displayed an ON/OFF ratio of about $10^{2}\left(V_{\mathrm{D}}=-0.6 \mathrm{~V}, V_{\mathrm{G}}=0.6 \mathrm{~V}\right)$ and a maximum transconductance of $6.0 \mathrm{mS}$ at $V_{\mathrm{G}}=0.1 \mathrm{~V}\left(V_{\mathrm{D}}=-0.6 \mathrm{~V}\right)$ at a time response in the range of $1 \mathrm{~min}(\tau \approx 55 \mathrm{~s})$. Thus, also the 
electrochemical response of PEDOT:Nafion was not drastically affected by the differences in processing.

In a final set of experiments, we aimed at illustrating that our 3D printing process can be used for the fabrication of complex architectures. To highlight the excellent printability of the material, we fabricated a conducting duckling, which we call a (con)duckling (Figure 4a), and a carbon nanotube-like structure (Figure 4b), which comprised details in the millimeter-range and could be printed with high precision. As a proof of concept, illustrating the potential for functional devices, we also $3 \mathrm{D}$ printed a set of thermoelectric modules. For organic materials, the optimal leg length is typically found in the millimeter range, ${ }^{12,62,63}$ a size that is difficult to realize with in-plane or $2 \mathrm{D}$ printed and folded modules but is perfectly suitable for FFF 3D printing. We chose to fabricate an out-ofplane thermoelectric module with a leg length of $1.6 \mathrm{~mm}$. The module was printed onto a textile substrate (a prewashed polyester fleece) and comprised 100 leg pairs (Figure 4c). In the first step, PEDOT:Nafion legs, which served as the p-type material, were prepared on top of the textile. In the second step, we printed interconnecting, pseudo n-type legs onto the PEDOT:Nafion structures using a commercial ABS/Carbon black composite which displays a Seebeck coefficient close to 0 $\left(\alpha_{\mathrm{ABS} / \mathrm{CB}}=1 \pm 1 \mu \mathrm{V} \mathrm{K}^{-1}, \sigma_{\mathrm{ABS} / \mathrm{CB}}=0.003 \mathrm{~S} \mathrm{~cm}^{-1}\right)$. In order to improve the thermoelectric performance of the module, the print was immersed into a base bath, ${ }^{64}$ which resulted in a slight dedoping of the PEDOT:Nafion and an increase of its Seebeck coefficient $\alpha_{\text {PEDOT:Nafion }}$ from 10 to $15 \pm 2 \mu \mathrm{V} \mathrm{K}^{-1}$ without a notable decrease of its conductivity. As expected, the thermoelectric properties of the ABS/carbon black composite were not affected by the base treatment.

To measure the thermoelectric performance, the module was then sandwiched between a hot-plate (textile side) and a heat sink, and the temperature difference $\Delta T_{\text {hot-cold }}$ between them was monitored by two thermocouples. The output voltage increased linearly from $9 \mathrm{mV}$ for $\Delta T_{\text {hot-cold }}=10^{\circ} \mathrm{C}$ to $39 \mathrm{mV}$ for $\Delta T_{\text {hot-cold }}=53{ }^{\circ} \mathrm{C}$ (Figure $\left.4 \mathrm{~d}\right)$. Thus, in the case of small temperature gradients, the obtained output voltage was close to the expected value of $V_{\text {out }} / \Delta T=N\left(\alpha_{\text {PEDOT:Nafion }}-\right.$ $\left.\alpha_{\mathrm{ABS} / \mathrm{CB}}\right)=1400 \mu \mathrm{V} \mathrm{K} \mathrm{K}^{-1}$, where $N$ is the number of leg pairs. Only at larger temperature gradients, the obtained voltage output started to deviate considerably from the estimated value. We explain this discrepancy with a non-negligible thermal contact resistance between the module and the heat source and sink, which reduces the temperature difference $\Delta T_{\text {leg }}$ that the thermoelectric legs experience (cf. Figure 4e; the measured and calculated power output match if $\Delta T_{\text {leg }} \approx$ $0.5 \cdot \Delta T_{\text {hot-cold }}$ is assumed). As a result of the low conductivity of the $\mathrm{ABS} /$ carbon black composite, the module displayed a considerable internal resistance $R_{\mathrm{i}}$ of about $400 \mathrm{k} \Omega$, which can be regarded as the main limiting factor for the power output of the device. We would like to point out that our module fabrication process is reproducible as evidenced by the very similar performance of a second, identical thermoelectric module (Figure 4d), affirming the robustness of our 3D printing method.

\section{CONCLUSIONS}

In conclusion, we have demonstrated an innovative strategy that enables the fabrication of flexible and conducting polymer structures via bulk processing using the polymer complex PEDOT:Nafion. Melt-spun PEDOT:Nafion fibers displayed conductivities of about $3 \mathrm{~S} \mathrm{~cm}^{-1}$, retained their conductivity (a)
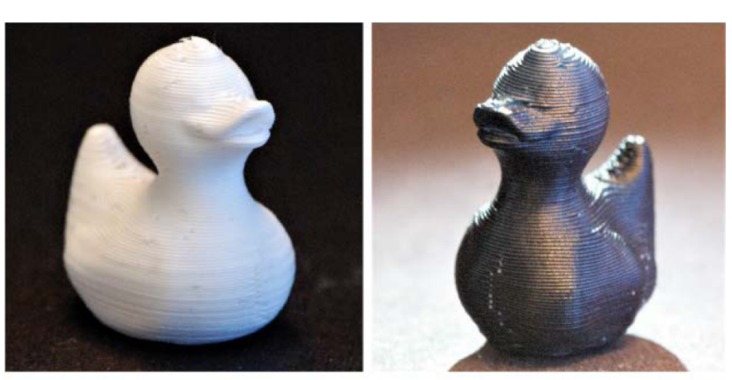

(b)
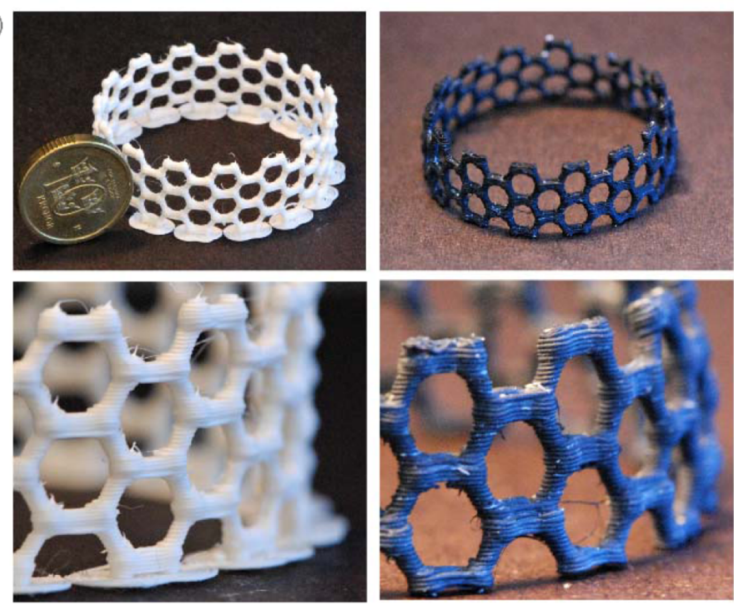

(c)

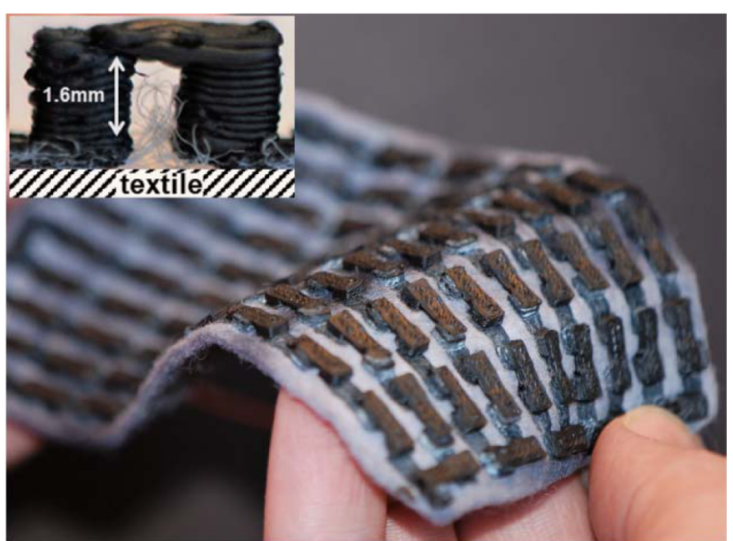

(d)

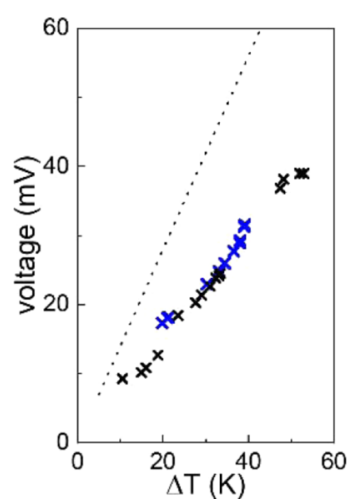

(e)

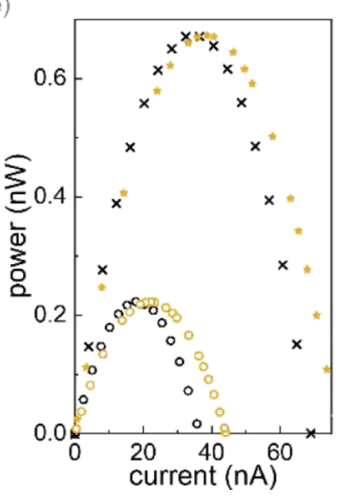

Figure 4. (a) FFF 3D printed, $1.6 \mathrm{~cm}$ tall Nafion/ABS duckling before (left) and after PEDOT synthesis (right). (b) Carbon nanotube-like structure before (left) and after PEDOT synthesis (right); the Swedish 10-krona coin has a diameter of $20.5 \mathrm{~mm}$. (c) 100-leg thermoelectric module (inset = single leg pair); (d) output voltage of two different (blue and black) 3D printed modules as a function of $\Delta T_{\text {hot-cold }}$ (the dotted line represents the theoretical output for $V_{\text {out }} / \Delta T=1400 \mu \mathrm{V} \mathrm{K} \mathrm{K}^{-1}$ ). (e) Experimentally measured power output for the module shown in (c) at $\Delta T_{\text {hot-cold }}=33 \mathrm{~K}$ (black circles) and $\Delta T_{\text {hot-cold }}=53 \mathrm{~K}$ (black crosses) and calculated power 
Figure 4. continued

output $P=\left(V_{\text {out }} / \Delta T \cdot \Delta T_{\mathrm{leg}}\right)^{2} \cdot R_{\mathrm{vl}} /\left(R_{\mathrm{i}}{ }^{2}+2 R_{\mathrm{i}} R_{\mathrm{vl}}+R_{\mathrm{vl}}{ }^{2}\right)$, where $R_{\mathrm{vl}}$ is the variable load resistance and $R_{\mathrm{i}}$ is the internal resistance and assuming that the legs experience a temperature difference $\Delta T_{\text {leg }}=14 \mathrm{~K}$ (yellow circles) and $\Delta T_{\text {leg }}=25 \mathrm{~K}$ (yellow stars).

upon stretching to more than $100 \%$ elongation, and were successfully integrated as a mixed ion-hole conductor in OECT devices. Furthermore, we developed a thermoplastic blend, which we used to $3 \mathrm{D}$ print conducting structures and figurines with millimeter-sized features. To highlight the potential for functional devices, we $3 \mathrm{D}$ printed an out-ofplane thermoelectric module with a leg length in the millimeter range, comprising 100 leg pairs with PEDOT:Nafion p-type legs connected via legs based on an ABS/Carbon black blend. The excellent mechanical properties together with the nontoxicity of the material and the ease of processing make bulk processed PEDOT:Nafion a promising candidate for wearable electronics, e-textiles, and biosensors.

\section{EXPERIMENTAL SECTION}

Materials. DMSO, $\mathrm{KOH}$ (>87\%), $\left(\mathrm{NH}_{4}\right)_{2} \mathrm{~S}_{2} \mathrm{O}_{8}, \mathrm{FeCl}_{3}$, and the ion exchange resin MP62 were purchased from Sigma-Aldrich and used as received. EDOT was purchased from TCI Europe and used without any further purification. The Nafion precursor 1100 was purchased from IonPower, Germany. Lewatit $\mathrm{S} 108 \mathrm{H}$ ion exchange resin was kindly provided by Lanxess, Germany.

Melt Processing. Melt spinning of the Nafion precursor was performed in an Xplore MC5 micro compounder together with an Xplore Micro Fiber Line. The Nafion precursor was compounded for $5 \mathrm{~min}$ at $240{ }^{\circ} \mathrm{C}$ and then extruded through a $0.5 \mathrm{~mm}$ nozzle. The extrudate was collected on the Xplore Micro Fiber Line, and spun into a fiber with a winding speed of $25 \mathrm{~m} \mathrm{~min}^{-1}$ and a winding torque of $50 \mathrm{Nm}$ and giving fibers with a thickness of $0.2 \mathrm{~mm}$. Blending of the Nafion precursor with $0,15,20$, and $30 \mathrm{wt} \%$ of $A B S$ was performed in an Xplore MC5 micro compounder for $5 \mathrm{~min}$ at $260{ }^{\circ} \mathrm{C}$ and then extruded through a $1.0 \mathrm{~mm}$ nozzle resulting in $1.75 \pm 0.1 \mathrm{~mm}$ filaments for FFF 3D printing.

Synthesis. To activate the Nafion precursor, the material was immersed into an alkaline bath of $\mathrm{H}_{2} \mathrm{O} / \mathrm{DMSO} / \mathrm{KOH}$ (50:35:15 wt $\%)$ under slight stirring at a temperature between 30 and $70{ }^{\circ} \mathrm{C}$ for 15 min to $4 \mathrm{~h}$, depending on the thickness of the activated layer that was aimed for. The activated Nafion was then washed with water and immersed into a water bath for at least $4 \mathrm{~h}$. To leach the ABS plastic from the Nafion/ABS blend, the material was immersed into a solution of $\mathrm{FeCl}_{3}$ in DMSO $\left(18 \mathrm{~g} \mathrm{~L}^{-1}\right)$ at $70{ }^{\circ} \mathrm{C}$ for 0.5 to $4 \mathrm{~h}$, depending on the thickness of the bulk structure and the thickness of the activated layer. For the synthesis of PEDOT onto Nafion, the EDOT monomer was dispersed in water $(17.5 \mathrm{mM})$ by tip sonication (2 s pulses, $100 \mathrm{~W}, 20 \mathrm{kHz}, 5 \mathrm{~min}$ ) until no droplets were visible to the eye and a slightly whitish dispersion was obtained. The Nafion bulk was immersed into the aqueous EDOT dispersion and upon addition of the oxidants $\left(\mathrm{NH}_{4}\right)_{2} \mathrm{~S}_{2} \mathrm{O}_{8}$ and $\mathrm{FeCl}_{3}$ (molar ratio EDOT/ $\left.\left(\mathrm{NH}_{4}\right)_{2} \mathrm{~S}_{2} \mathrm{O}_{8} / \mathrm{FeCl}_{3}=1: 1: 0.3\right)$, the dispersion was left for polymerization at room temperature and under ambient conditions for $50 \mathrm{~h}$. For the synthesis of PEDOT onto Nafion/ABS, the leached, ABS poor structure was immersed into an aqueous dispersion of EDOT in water $\left(1.875 \mathrm{~mL} \mathrm{~L}^{-1}\right)$, prepared by tip sonication. $\left(\mathrm{NH}_{4}\right)_{2} \mathrm{~S}_{2} \mathrm{O}_{8}$ was added as an oxidant (molar ratio EDOT/ $\left(\mathrm{NH}_{4}\right)_{2} \mathrm{~S}_{2} \mathrm{O}_{8}=1: 1$ ), and the polymerization proceeded at room temperature and under ambient conditions for $50 \mathrm{~h}$. For purification, the PEDOT:Nafion bulk was immersed overnight into a water bath containing an excess of the acidic cation exchange resin Lewatit MonoPlus S108H and the anion exchange resin Lewatit MP 62.

FFF 3D Printing. FFF of the extruded Nafion/ABS filament was performed with a Massportal Pharaoh XD20, with a nozzle temperature of $260{ }^{\circ} \mathrm{C}$, a printing surface with a temperature of $110^{\circ} \mathrm{C}$, and a printing speed of $1000 \mathrm{~mm} \mathrm{~min}^{-1}$. As a textile substrate for the thermoelectric module, we used a polyester fleece, which was prewashed in $\mathrm{H}_{2} \mathrm{O} / \mathrm{DMSO} / \mathrm{KOH}$ (50:35:15 wt \%) to avoid shrinkage during the activation step.

Mechanical Testing. Tensile testing of PEDOT:Nafion fibers was performed using a DMA Q800 (TA Instruments) with a ramped force of $0.5 \mathrm{~N} \mathrm{~min}^{-1}$. The dynamic characterization was performed by subjecting a fiber to 100 cycles of $6 \mathrm{~s}$ displacement to $10 \%$ strain followed by $30 \mathrm{~s}$ release. The electrical resistance was measured in situ using a Keysight U1253B multimeter collecting 1 data point $\mathrm{s}^{-1}$. Samples of the Nafion/ABS blends for tensile testing were prepared in a hot press (AB Nike Hydralik) at $240{ }^{\circ} \mathrm{C}$ in two steps, 5 ton for 2 $\mathrm{min}$ and 10 tons for $3 \mathrm{~min}$, with $0.1 \mathrm{~mm}$ spacers. The resulting films were cut into strips with a width of $4 \mathrm{~mm}$ and a thickness varying from 0.07 to $0.13 \mathrm{~mm}$. The Young's modulus of Nafion/ABS blends was determined from tensile tests at a force rate of $1 \mathrm{~N} \mathrm{~min}^{-1}$ using a DMA Q800 (TA Instruments). To test the adhesion between Nafion/ ABS blends, ABS and HIPS, rectangular test strips $(30 \mathrm{~mm} \times 5 \mathrm{~mm} \times$ $0.2 \mathrm{~mm}$ ) were 3D printed onto printed substrates (thickness $0.2 \mathrm{~mm}$ ). At one end, the strips were separated from the substrate by a thin paper. The loose ends of the strips and the substrate were mounted into a DMA TA Q800, and peeling tests were performed with a

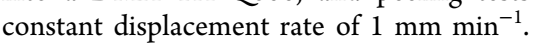

Electrical Characterization. To determine the conductivity of the material, we measured the resistance of PEDOT:Nafion filaments with different thicknesses and of printed lines/sheets with different dimensions. PEDOT:Nafion filaments and printed lines were contacted with Ag paste (Agar Scientific) in two-point configuration (channel length from 2 to $15 \mathrm{~mm}$ ), and current-voltage characteristics were recorded with a Keithley 2400 source/measure unit (SMU). To calculate the electrical conductivity, the dimensions of the PEDOT:Nafion composite were determined using an optical microscope. Because the conductivity of the material did not vary as a function of the channel length or dimension of the sample, but only as a function of the synthesis conditions, we assume that the material's conductivity within the complex 3D structures is comparable to the conductivity of the material printed in a straight line or sheets.

OECTs were prepared by coating parts of PEDOT:Nafion fibers and printed PEDOT:Nafion lines with silver paste to create electrodes. The electrodes were then encapsulated using a melt-glue gun with polyurethane glue (Dana Lim A/S). The devices were dipped in a $0.1 \mathrm{M} \mathrm{NaCl}$ electrolyte together with an $\mathrm{Ag} / \mathrm{AgCl}$ gate electrode, and the IV characteristics were measured using two Keithley 2400 SMUs and customized LabVIEW and Matlab software. The Seebeck coefficient of PEDOT:Nafion filaments was measured at $300 \mathrm{~K}$ using a SB1000 instrument from MMR Technologies equipped with a K2000 temperature controller at a thermal load of $1-2 \mathrm{~K}$ and a constantan wire as an internal reference. For thermoelectric characterization, the printed module was sandwiched between a variable hot plate and a thin metal plate with an ice pack on top. The hot and cold temperatures were registered using two surface mounted K-type thermocouples (Omega engineering). For proper electrical contact, two copper wires were connected to the module nodes using silver paste. The output voltage was measured using a Keithley 2400 source meter which also functioned as a variable load by sourcing current. The setup was controlled, and data was recorded via a customized LabVIEW software.

Impedance Spectroscopy. Impedance spectroscopy was performed in a $0.1 \mathrm{M} \mathrm{NaCl}$ electrolyte using a $\mathrm{CHI} 650 \mathrm{~d}(\mathrm{CH}$ Instruments) chemical work station. A frequency range from $10^{-2}$ to $10^{5} \mathrm{~Hz}$ was scanned. The OECT samples with shortened source and drain electrodes were used as the working electrode, a Pt wire (diameter $1 \mathrm{~mm}$ ) as the counter electrode, and an $\mathrm{Ag} / \mathrm{AgCl}$ pellet as the reference electrode.

Scanning Electron Microscopy. SEM was performed using a JEOL JSM-7800F Prime instrument with an acceleration voltage of 3 $\mathrm{kV}$. 
X-ray Scattering. SAXS diffractograms were obtained using a Mat:Nordic (SAXSLAB) equipped with a Rigaku 003+ high brilliance micro focus Cu-radiation source (wavelength $=1.5406 \AA$ ) and a Pilatus $300 \mathrm{~K}$ detector placed at a distance of $1050 \mathrm{~mm}$ from the sample. WAXS images were obtained at the D-line at the Cornell High Energy Synchrotron Source (CHESS) at Cornell University. The measurements were performed with a synchrotron radiation of a wavelength of $1.155 \AA$. A Pilatus $200 \mathrm{~K}$ detector with pixel size of 172 $\mu \mathrm{m} \times 172 \mu \mathrm{m}$ was used to collect scattered X-ray from the sample at a sample to detector distance of $177.2 \mathrm{~mm}$.

Elemental Analysis. Elemental analysis was performed using inductively coupled plasma-mass spectroscopy on dried samples (60 ${ }^{\circ} \mathrm{C},>4 \mathrm{~h}$ ) at Mikrolab Kolbe, Germany.

Attenuated Total Reflection-Fourier Transform Infrared Spectroscopy. Infrared absorption measurements were performed on bulk samples (dimensions ca. $2 \mathrm{~mm} \times 5 \mathrm{~mm} \times 1 \mathrm{~mm}$ ) using a Frontier FTIR Spectrometer from PerkinElmer equipped with a GladiATR attachment from Pike Technologies.

\section{ASSOCIATED CONTENT}

\section{(s) Supporting Information}

The Supporting Information is available free of charge at https://pubs.acs.org/doi/10.1021/acsami.9b20615.

FTIR absorption spectra, elemental analysis, WAXS and SAXS diffractograms, mechanical properties of PEDOT:Nafion fibers, water uptake of PEDOT:Nafion, capacitance measurements, scanning electron micrographs, and OECT device characterization (PDF)

\section{AUTHOR INFORMATION}

\section{Corresponding Authors}

Anna I. Hofmann - Department of Chemistry and Chemical Engineering, Chalmers University of Technology, 41296 Göteborg, Sweden; 이이이.0rg/0000-0002-4480-6028; Email: hofanna@chalmers.se

Christian Müller - Department of Chemistry and Chemical Engineering, Chalmers University of Technology, 41296 Göteborg, Sweden; 이이. orcid.org/0000-0001-7859-7909; Email: christian.muller@chalmers.se

\section{Authors}

Ida Östergren - Department of Chemistry and Chemical Engineering, Chalmers University of Technology, 41296 Göteborg, Sweden

Youngseok Kim - School of Materials Science and Engineering, Gwangiu Institute of Science and Technology, 61005 Gwangju, Republic of Korea

Sven Fauth - Department of Chemistry and Chemical Engineering, Chalmers University of Technology, 41296 Göteborg, Sweden

Mariavittoria Craighero - Department of Chemistry and Chemical Engineering, Chalmers University of Technology, 41296 Göteborg, Sweden

Myung-Han Yoon - School of Materials Science and Engineering, Gwangju Institute of Science and Technology, 61005 Gwangju, Republic of Korea; 10 orcid.org/0000-00017205-3054

Anja Lund - Department of Chemistry and Chemical Engineering, Chalmers University of Technology, 41296 Göteborg, Sweden

Complete contact information is available at: https://pubs.acs.org/10.1021/acsami.9b20615

\section{Author Contributions}

${ }^{\S}$ A.I.H. and I.Ö. contributed equally.

\section{Notes}

The authors declare no competing financial interest.

\section{ACKNOWLEDGMENTS}

We gratefully acknowledge financial support from the Swedish Research Council through grant no. 2016-06146, the Swedish Foundation for Strategic Research through grant no. RMA 150052, the Knut and Alice Wallenberg Foundation through a Wallenberg Academy Fellowship, and the European Research Council (ERC) under grant agreement no. 637624. Furthermore, we thank the Cornell High Energy Synchrotron Source (CHESS) (supported by the NSF \& NIH/NIGMS via NSF award DMR-1332208) for providing experimental time for WAXS measurements. SAXS measurements and electron microscopy were performed at the Chalmers Material Analysis Laboratory (CMAL). Furthermore, we thank Dr. Hengda Sun and Dr. Simone Fabiano from Linköping University for discussions regarding the design of bulk OECTs, and Lanxess for providing the Lewatit $\mathrm{S} 108 \mathrm{H}$ ion exchange resin.

\section{REFERENCES}

(1) Kroon, R.; Mengistie, D. A.; Kiefer, D.; Hynynen, J.; Ryan, J. D.; Yu, L.; Müller, C. Thermoelectric Plastics: From Design to Synthesis, Processing and Structure-Property Relationships. Chem. Soc. Rev. 2016, 45, 6147-6164.

(2) Russ, B.; Glaudell, A.; Urban, J. J.; Chabinyc, M. L.; Segalman, R. A. Organic Thermoelectric Materials for Energy Harvesting and Temperature Control. Nat. Rev. Mater. 2016, 1, 16050.

(3) Seyedin, S.; Razal, J. M.; Innis, P. C.; Jeiranikhameneh, A.; Beirne, S.; Wallace, G. G. Knitted Strain Sensor Textiles of Highly Conductive All-Polymeric Fibers. ACS Appl. Mater. Interfaces 2015, 7, 21150-21158.

(4) Stoppa, M.; Chiolerio, A. Wearable Electronics and Smart Textiles: A Critical Review. Sensors 2014, 14, 11957-11992.

(5) Lund, A.; van der Velden, N. M.; Persson, N.-K.; Hamedi, M. M.; Müller, C. Electrically Conducting Fibres for E-Textiles: An Open Playground for Conjugated Polymers and Carbon Nanomaterials. Mater. Sci. Eng., R 2018, 126, 1-29.

(6) Wang, B.; Facchetti, A. Mechanically Flexible Conductors for Stretchable and Wearable E-Skin and E-Textile Devices. Adv. Mater. 2019, 31, 1901408.

(7) Matsuhisa, N.; Chen, X.; Bao, Z.; Someya, T. Materials and Structural Designs of Stretchable Conductors. Chem. Soc. Rev. 2019, 48, 2946-2966.

(8) Berggren, M.; Richter-Dahlfors, A. Organic Bioelectronics. Adv. Mater. 2007, 19, 3201-3213.

(9) Rivnay, J.; Inal, S.; Salleo, A.; Owens, R. M.; Berggren, M.; Malliaras, G. G. Organic Electrochemical Transistors. Nat. Rev. Mater. 2018, 3, 17086.

(10) Bubnova, O.; Crispin, X. Towards Polymer-Based Organic Thermoelectric Generators. Energy Environ. Sci. 2012, 5, 9345-9362.

(11) Mayer, P. M.; Ram, R. J. Optimization of Heat Sink-Limited Thermoelectric Generators. Nanoscale Microscale Thermophys. Eng. 2006, 10, 143-155.

(12) Ferhat, S.; Domain, C.; Vidal, J.; Noël, D.; Ratier, B.; Lucas, B. Organic Thermoelectric Devices Based on a Stable N-Type Nanocomposite Printed on Paper. Sustainable Energy Fuels 2018, 2, 199-208.

(13) Beretta, D.; Perego, A.; Lanzani, G.; Caironi, M. Organic Flexible Thermoelectric Generators: From Modeling, a Roadmap towards Applications. Sustainable Energy Fuels 2017, 1, 174-190.

(14) Hynynen, J.; Järsvall, E.; Kroon, R.; Zhang, Y.; Barlow, S.; Marder, S. R.; Kemerink, M.; Lund, A.; Müller, C. Enhanced 
Thermoelectric Power Factor of Tensile Drawn Poly(3-Hexylthiophene). ACS Macro Lett. 2019, 8, 70-76.

(15) Brandão, L.; Viana, J.; Bucknall, D. G.; Bernardo, G. Solventless Processing of Conjugated Polymers - A Review. Synth. Met. 2014, 197, 23-33.

(16) O'Carroll, D.; Lieberwirth, I.; Redmond, G. Melt-Processed Polyfluorene Nanowires as Active Waveguides. Small 2007, 3, 11781183.

(17) Kroon, R.; Ryan, J. D.; Kiefer, D.; Yu, L.; Hynynen, J.; Olsson, E.; Müller, C. Bulk Doping of Millimeter-Thick Conjugated Polymer Foams for Plastic Thermoelectrics. Adv. Funct. Mater. 2017, 27, 1704183.

(18) Holness, F. B.; Price, A. D. Direct Ink Writing of 3D Conductive Polyaniline Structures and Rheological Modelling. Smart Mater. Struct. 2018, 27, 015006.

(19) Titelman, G. I.; Zilberman, M.; Siegmann, A.; Haba, Y.; Narkis, M. Thermal Dynamic Processing of Polyaniline with Dodecylbenzene Sulfonic Acid. J. Appl. Polym. Sci. 1997, 66, 2199-2208.

(20) Cao, Y.; Smith, P.; Heeger, A. J. Counter-Ion Induced Processibility of Conducting Polyaniline and of Conducting Polyblends of Polyaniline in Bulk Polymers. Synth. Met. 1992, 48, 91-97.

(21) Drozda, T.; Wick, C.; Benedict, J. T.; Veilleux, R. F.; Bakerjian, R. Tool and Manufacturing Engineers Handbook: A Reference Book for Manufacturing Engineers, Managers, and Technicians; Society of Manufacturing Engineers, 1983.

(22) Jacobs, I. E.; Moulé, A. J. Controlling Molecular Doping in Organic Semiconductors. Adv. Mater. 2017, 29, 1703063.

(23) Green, R. A.; Lovell, N. H.; Wallace, G. G.; Poole-Warren, L. A. Conducting Polymers for Neural Interfaces: Challenges in Developing an Effective Long-Term Implant. Biomaterials 2008, 29, 3393-3399.

(24) Njuguna, J.; Ansari, F.; Sachse, S.; Zhu, H.; Rodriguez, V. M. Nanomaterials, Nanofillers, and Nanocomposites: Types and Properties. In Health and Environmental Safety of Nanomaterials: Polymer Nanocomposites and Other Materials Containing Nanoparticles; Njuguna, J., Pielichowski, K., Zhu, H., Eds.; Woodhead Publishing, 2014; pp 3-27.

(25) Elschner, A.; Kirchmeyer, S.; Lovenich, W.; Merker, U.; Reuter, K. PEDOT: Principles and Applications of an Intrinsically Conductive Polymer; CRC Press, 2010.

(26) Richardson-Burns, S. M.; Hendricks, J. L.; Foster, B.; Povlich, L. K.; Kim, D.-H.; Martin, D. C. Polymerization of the Conducting Polymer Poly(3,4-Ethylenedioxythiophene) (PEDOT) around Living Neural Cells. Biomaterials 2007, 28, 1539-1552.

(27) Fujie, T. Development of Free-Standing Polymer Nanosheets for Advanced Medical and Health-Care Applications. Polym. J. 2016, $48,773-780$.

(28) Greco, F.; Zucca, A.; Taccola, S.; Menciassi, A.; Fujie, T.; Haniuda, H.; Takeoka, S.; Dario, P.; Mattoli, V. Ultra-Thin Conductive Free-Standing PEDOT/PSS Nanofilms. Soft Matter 2011, 7, 10642-10650.

(29) Kee, S.; Kim, H.; Paleti, S. H. K.; El Labban, A.; Neophytou, M.; Emwas, A.-H.; Alshareef, H. N.; Baran, D. Highly Stretchable and Air-Stable PEDOT:PSS/Ionic Liquid Composites for Efficient Organic Thermoelectrics. Chem. Mater. 2019, 31, 3519-3526.

(30) Kim, Y.; Lim, T.; Kim, C.-H.; Yeo, C. S.; Seo, K.; Kim, S.-M.; Kim, J.; Park, S. Y.; Ju, S.; Yoon, M.-H. Organic Electrochemical Transistor-Based Channel Dimension-Independent Single-Strand Wearable Sweat Sensors. NPG Asia Mater. 2018, 10, 1086-1095.

(31) Jalili, R.; Razal, J. M.; Innis, P. C.; Wallace, G. G. One-Step Wet-Spinning Process of Poly(3,4-Ethylenedioxythiophene):Poly(Styrenesulfonate) Fibers and the Origin of Higher Electrical Conductivity. Adv. Funct. Mater. 2011, 21, 3363-3370.

(32) Liu, J.; Jia, Y.; Jiang, Q.; Jiang, F.; Li, C.; Wang, X.; Liu, P.; Liu, P.; Hu, F.; Du, Y.; et al. Highly Conductive Hydrogel Polymer Fibers toward Promising Wearable Thermoelectric Energy Harvesting. ACS Appl. Mater. Interfaces 2018, 10, 44033-44040.

(33) Zhou, J.; Li, E. Q.; Li, R.; Xu, X.; Ventura, I. A.; Moussawi, A.; Anjum, D. H.; Hedhili, M. N.; Smilgies, D.-M.; Lubineau, G.; et al.
Semi-Metallic, Strong and Stretchable Wet-Spun Conjugated Polymer Microfibers. J. Mater. Chem. C 2015, 3, 2528-2538.

(34) Zhang, J.; Seyedin, S.; Qin, S.; Lynch, P. A.; Wang, Z.; Yang, W.; Wang, X.; Razal, J. M. Fast and Scalable Wet-Spinning of Highly Conductive PEDOT:PSS Fibers Enables Versatile Applications. J. Mater. Chem. A 2019, 7, 6401-6410.

(35) Sarabia-Riquelme, R.; Shahi, M.; Brill, J. W.; Weisenberger, M. C. Effect of Drawing on the Electrical, Thermoelectrical, and Mechanical Properties of Wet-Spun PEDOT:PSS Fibers. ACS Appl. Polym. Mater. 2019, 1, 2157-2167.

(36) Hansen, T. S.; West, K.; Hassager, O.; Larsen, N. B. Highly Stretchable and Conductive Polymer Material Made from Poly(3,4Ethylenedioxythiophene) and Polyurethane Elastomers. Adv. Funct. Mater. 2007, 17, 3069-3073.

(37) Taroni, P. J.; Santagiuliana, G.; Wan, K.; Calado, P.; Qiu, M.; Zhang, H.; Pugno, N. M.; Palma, M.; Stingelin-Stutzman, N.; Heeney, M.; et al. Toward Stretchable Self-Powered Sensors Based on the Thermoelectric Response of PEDOT:PSS/Polyurethane Blends. Adv. Funct. Mater. 2018, 28, 1704285.

(38) Seyedin, M. Z.; Razal, J. M.; Innis, P. C.; Wallace, G. G. StrainResponsive Polyurethane/PEDOT:PSS Elastomeric Composite Fibers with High Electrical Conductivity. Adv. Funct. Mater. 2014, 24, 2957-2966.

(39) Hofmann, A. I.; Smaal, W. T. T.; Mumtaz, M.; Katsigiannopoulos, D.; Brochon, C.; Schütze, F.; Hild, O. R.; Cloutet, E.; Hadziioannou, G. An Alternative Anionic Polyelectrolyte for Aqueous PEDOT Dispersions: Toward Printable Transparent Electrodes. Angew. Chem., Int. Ed. 2015, 54, 8506-8510.

(40) Sanchez-Sanchez, A.; del Agua, I.; Malliaras, G. G.; Mecerreyes, D. Conductive Poly(3,4-Ethylenedioxythiophene) (PEDOT)-Based Polymers and Their Applications in Bioelectronics. In Smart Polymers and Their Applications; Aguilar, M. R., San Román, J., Eds.; Woodhead Publishing, 2019; pp 191-218.

(41) Hofmann, A. I.; Katsigiannopoulos, D.; Mumtaz, M.; Petsagkourakis, I.; Pecastaings, G.; Fleury, G.; Schatz, C.; Pavlopoulou, E.; Brochon, C.; Hadziioannou, G.; et al. How to Choose Polyelectrolytes for Aqueous Dispersions of Conducting PEDOT Complexes. Macromolecules 2017, 50, 1959-1969.

(42) del Agua, I.; Mantione, D.; Casado, N.; Sanchez-Sanchez, A.; Malliaras, G. G.; Mecerreyes, D. Conducting Polymer Iongels Based on PEDOT and Guar Gum. ACS Macro Lett. 2017, 6, 473-478.

(43) Wang, W.; Cui, M.; Song, Z.; Luo, X. An Antifouling Electrochemical Immunosensor for Carcinoembryonic Antigen Based on Hyaluronic Acid Doped Conducting Polymer PEDOT. RSC Adv. 2016, 6, 88411-88416.

(44) Vreeland, R. F.; Atcherley, C. W.; Russell, W. S.; Xie, J. Y.; Lu, D.; Laude, N. D.; Porreca, F.; Heien, M. L. Biocompatible PEDOT:Nafion Composite Electrode Coatings for Selective Detection of Neurotransmitters in Vivo. Anal. Chem. 2015, 87, 2600-2607.

(45) Wang, P.; Olbricht, W. L. PEDOT/Nafion Composite Thin Films Supported on Pt Electrodes: Facile Fabrication and Electrochemical Activities. Chem. Eng. J. 2010, 160, 383-390.

(46) Li, L.; Drillet, J.-F.; Dittmeyer, R.; Jüttner, K. Formation and Characterization of PEDOT-Modified Nafion 117 Membranes. J. Solid State Electrochem. 2006, 10, 708-713.

(47) Higgins, S. J.; Lovell, K. V.; Gamini Rajapakse, R. M.; Walsby, N. M. Grafting and Electrochemical Characterisation of Poly-(3,4Ethylenedioxythiophene) Films, on Nafion and on Radiation-Grafted Polystyrenesulfonate-Polyvinylidene Fluoride Composite Surfaces. J. Mater. Chem. 2003, 13, 2485-2489.

(48) Carli, S.; Bianchi, M.; Zucchini, E.; Di Lauro, M.; Prato, M.; Murgia, M.; Fadiga, L.; Biscarini, F. Electrodeposited PEDOT:Nafion Composite for Neural Recording and Stimulation. Adv. Healthcare Mater. 2019, 8, 1900765.

(49) Heitner-Wirguin, C. Recent Advances in Perfluorinated Ionomer Membranes: Structure, Properties and Applications. J. Membr. Sci. 1996, 120, 1-33. 
(50) Carrico, J. D.; Traeden, N. W.; Aureli, M.; Leang, K. K. Fused Filament 3D Printing of Ionic Polymer-Metal Composites (IPMCs). Smart Mater. Struct. 2015, 24, 125021.

(51) Elliott, J. A.; James, P. J.; McMaster, T. J.; Newton, J. M.; Elliott, A. M. S.; Hanna, S.; Miles, M. J. Hydrolysis of the Nafion Precursor Studied by X-Ray Scattering and in-Situ Atomic Force Microscopy. E-Polymers 2001, 1, 022.

(52) Salerno, H. L. S.; Beyer, F. L.; Elabd, Y. A. Anion Exchange Membranes Derived from Nafion Precursor for the Alkaline Fuel Cell. J. Polym. Sci., Part B: Polym. Phys. 2012, 50, 552-562.

(53) Mauritz, K. A.; Moore, R. B. State of Understanding of Nafion. Chem. Rev. 2004, 104, 4535-4586.

(54) Crispin, X.; Jakobsson, F. L. E.; Crispin, A.; Grim, P. C. M.; Andersson, P.; Volodin, A.; van Haesendonck, C.; Van der Auweraer, M.; Salaneck, W. R.; Berggren, M. The Origin of the High Conductivity of Poly(3,4-Ethylenedioxythiophene)-Poly(Styrenesulfonate) (PEDOT-PSS) Plastic Electrodes. Chem. Mater. 2006, 18, 4354-4360.

(55) Elliott, J. A.; Wu, D.; Paddison, S. J.; Moore, R. B. A Unified Morphological Description of Nafion Membranes from SAXS and Mesoscale Simulations. Soft Matter 2011, 7, 6820-6827.

(56) Haubold, H.-G.; Vad, T.; Jungbluth, H.; Hiller, P. Nano Structure of NAFION: A SAXS Study. Electrochim. Acta 2001, 46, $1559-1563$.

(57) Gruger, A.; Régis, A.; Schmatko, T.; Colomban, P. Nanostructure of Nafion Membranes at Different States of Hydration: An IR and Raman Study. Vib. Spectrosc. 2001, 26, 215-225.

(58) Musumeci, C.; Vagin, M.; Zeglio, E.; Ouyang, L.; Gabrielsson, R.; Inganäs, O. Organic Electrochemical Transistors from Supramolecular Complexes of Conjugated Polyelectrolyte PEDOTS. J. Mater. Chem. C 2019, 7, 2987-2993.

(59) Kim, S.-M.; Kim, C.-H.; Kim, Y.; Kim, N.; Lee, W.-J.; Lee, E.H.; Kim, D.; Park, S.; Lee, K.; Rivnay, J.; et al. Influence of PEDOT:PSS Crystallinity and Composition on Electrochemical Transistor Performance and Long-Term Stability. Nat. Commun. 2018, 9, 3858.

(60) Proctor, C. M.; Rivnay, J.; Malliaras, G. G. Understanding Volumetric Capacitance in Conducting Polymers. J. Polym. Sci., Part B: Polym. Phys. 2016, 54, 1433-1436.

(61) Rivnay, J.; Leleux, P.; Ferro, M.; Sessolo, M.; Williamson, A.; Koutsouras, D. A.; Khodagholy, D.; Ramuz, M.; Strakosas, X.; Owens, R. M.; et al. High-Performance Transistors for Bioelectronics through Tuning of Channel Thickness. Sci. Adv. 2015, 1, No. e1400251.

(62) Beretta, D.; Neophytou, N.; Hodges, J. M.; Kanatzidis, M. G.; Narducci, D.; Martin- Gonzalez, M.; Beekman, M.; Balke, B.; Cerretti, G.; Tremel, W.; et al. Thermoelectrics: From History, a Window to the Future. Mater. Sci. Eng., R 2019, 138, 100501.

(63) Bahk, J.-H.; Fang, H.; Yazawa, K.; Shakouri, A. Flexible Thermoelectric Materials and Device Optimization for Wearable Energy Harvesting. J. Mater. Chem. C 2015, 3, 10362-10374.

(64) Fan, Z.; Li, P.; Du, D.; Ouyang, J. Significantly Enhanced Thermoelectric Properties of PEDOT:PSS Films through Sequential Post-Treatments with Common Acids and Bases. Adv. Energy Mater. 2017, 7, 1602116. 